University of Nebraska - Lincoln

DigitalCommons@University of Nebraska - Lincoln

$2-15-2000$

\title{
Phase transition in the surface structure in copolymer films of vinylidene fluoride (70\%) with trifluoroethylene (30\%)
}

Jaewu Choi

University of Nebraska-Lincoln, jchoi@ece.eng.wayne.edu

C.N. Borca

University of Nebraska-Lincoln

Peter A. Dowben

University of Nebraska-Lincoln, pdowben@unl.edu

A. Bune

University of Nebraska-Lincoln

Matt Poulsen

University of Nebraska-Lincoln, map@suiter.com

See next page for additional authors

Follow this and additional works at: https://digitalcommons.unl.edu/physicsdowben

Part of the Physics Commons

Choi, Jaewu; Borca, C.N.; Dowben, Peter A.; Bune, A.; Poulsen, Matt; Pebley, Shane; Adenwalla, Shireen; Ducharme, Stephen; Robertson, Lee; Fridkin, V.M.; Petukhova, N.N.; and Yudin, S.G., "Phase transition in the surface structure in copolymer films of vinylidene fluoride (70\%) with trifluoroethylene (30\%)" (2000). Peter Dowben Publications. 33.

https://digitalcommons.unl.edu/physicsdowben/33

This Article is brought to you for free and open access by the Research Papers in Physics and Astronomy at DigitalCommons@University of Nebraska - Lincoln. It has been accepted for inclusion in Peter Dowben Publications by an authorized administrator of DigitalCommons@University of Nebraska - Lincoln. 


\section{Authors}

Jaewu Choi, C.N. Borca, Peter A. Dowben, A. Bune, Matt Poulsen, Shane Pebley, Shireen Adenwalla, Stephen Ducharme, Lee Robertson, V.M. Fridkin, N.N. Petukhova, and S.G. Yudin 


\title{
Phase transition in the surface structure in copolymer films of vinylidene fluoride (70\%) with trifluoroethylene $(30 \%)$
}

\author{
Jaewu Choi, C. N. Borca, P. A. Dowben, A. Bune, M. Poulsen, Shawn Pebley, S. Adenwalla, and Stephen Ducharme \\ Department of Physics and Astronomy, Behlen Laboratory of Physics, Center for Materials Research and Analysis, \\ University of Nebraska-Lincoln, Lincoln, Nebraska 68588-0111 \\ Lee Robertson \\ High Flux Isotope Reactor (HFIR), Oak Ridge National Laboratory, Oak Ridge, Tennessee 37831 \\ V. M. Fridkin, S. P. Palto, N. N. Petukhova, and S. G. Yudin \\ Institute of Crystallography, The Russian Academy of Science, 117333 Moscow, Russia \\ (Received 16 November 1998; revised manuscript received 30 March 1999)
}

\begin{abstract}
Surface structures and a surface structure phase transition are identified that are distinct from the known bulk ferroelectric-paraelectric phase transition of crystalline copolymer films of vinylidene fluoride (70\%) with trifluoroethylene $(30 \%)$. The temperature-dependent changes in the surface structure are accompanied by the physical rotation of the polar group $\left(\mathrm{CH}_{2}-\mathrm{CF}_{2}\right)$. These changes in the surface structure are compared to the bulk phase transition. We show that the bulk structural transition, while distinct from the surface, is qualitatively similar in both thick and thin Langmuir-Blodgett-grown films.
\end{abstract}

\section{INTRODUCTION}

Surface structural transitions, accompanied and likely driven by changes in band structure, are well known for metal surfaces. ${ }^{1}$ Among the best characterized examples of such surface reconstructions are $\mathrm{Mo}(100),{ }^{2,3} \mathrm{~W}(100),{ }^{3,4}$ and Mo(112). ${ }^{5}$ These examples appear to be mediated by bands that cross or closely approach the Fermi level. Ferroelectric transitions have long been anticipated to drive changes in band structure as well, ${ }^{6}$ so a surface ferroelectric transition, distinct from the bulk, could accompany a surface structural phase transition that is distinct from bulk.

Recently, a surface ferroelectric phase transition has been identified in ultrathin copolymer films of polyvinylidene fluoride with trifluoroethylene $\left(\mathrm{CH}_{2}-\mathrm{CF}_{2}: \mathrm{CHF}-\mathrm{CF}_{2}, 70 \%: 30 \%\right){ }^{7}$ The surface ferroelectric transition at $20^{\circ} \mathrm{C}$ is distinct from the bulk ferroelectric to paraelectric phase transition at about $80^{\circ} \mathrm{C}$, and is accompanied by a change in metallicity ${ }^{8}$ and a doubling of the surface Brillouin-zone size. ${ }^{9}$ While surface phase transitions, associated with changes in the electronic structure and metallicity, may be associated with a host of critical phenomena, ${ }^{10,11}$ the ultrathin copolymer films are the first ferroelectrics to show such connections.

The bulk ferroelectric-paraelectric phase transition in polyvinylidene fluoride (PVDF) with trifluoroethylene (TrFE) has long been associated with bulk structural changes and the appearances of piezoelectric and pyroelectric effects. ${ }^{12,13}$ Several distinct bulk crystal structures of ferroelectric PVDF and P(VDF-TrFE) have been postulated. ${ }^{12-19}$ The simplest conformational structure is formed in the ferroelectric $\beta$ phase, where the polymer chains have all-trans conformation and, each monomer $\left(\mathrm{CH}_{2}-\mathrm{CF}_{2}\right)$ is aligned roughly perpendicular to the polymer chain and has its own permanent dipole moment, directed from the negative fluorine to the positive hydrogen. The ferroelectric $\beta$ phase is usually observed below a ferroelectric Curie temperature (that is higher for higher VDF content) following poling with a high electric field and mechanical drawing to convert the chain conformation from the nonpolar $\alpha$ phase. $^{13,14,20}$ The $\alpha$ phase consists of an alternating trans $(T)$ gauche $(G)$ bonding configuration (or TGT $\bar{G}$ pattern) arranged in a nonpolar, paraelectric, structure. ${ }^{13,14,20}$ The weakly polar $\delta$ and $\gamma$ phases and other nonpolar phases (particularly the helical phase of polyethylene and other hydrogen-rich compositions) are not known or expected to take part in a ferroelectric transition. ${ }^{12-22}$

Surface structures can be different from bulk even if compositionally the same. This is true of both the clean semiconductor surfaces that exhibit surface structural transitions related to the surface nonmetal to metal transition that occur with increasing temperature ${ }^{11}$ and the surface reconstructions in metals that undergo a surface-temperature-dependent structural transformation, as noted for $\operatorname{Mo}(100),{ }^{2} \operatorname{Mo}(112),{ }^{5}$ and $\mathrm{W}(100) .{ }^{4}$ In this work, we show that the crystalline copolymer $\mathrm{P}(\mathrm{VDF}-\mathrm{TrFE}$ 70:30) has a reconstructed surface structure and undergoes a surface structural phase transition across the surface ferroelectric phase transition. ${ }^{7-9}$

\section{SAMPLE PREPARATION AND EXPERIMENTAL DETAILS}

The films of the P(VDF-TrFE, 70:30 mol\%) copolymer were prepared using a horizontal Langmuir-Blodgett method as described elsewhere. ${ }^{23,24}$ The films were deposited on silicon (111) for photoemission studies, on an aluminum-coated glass substrate with a sputtered aluminum top electrode for dielectric studies, and on cleaved graphite for scanning tunneling microscope studies.

The angle-resolved inverse photoemission spectroscopy (ARIPES), angle-resolved x-ray core-level photoemission spectroscopy, low-energy electron diffraction (LEED), and work-function measurements from the energy of the cutoff edge of the secondary electron in the photoemission were 
performed in the same ultrahigh-vacuum chamber with a base pressure better than $2 \times 10^{-10}$ Torr. The angle-resolved $\mathrm{X}$-ray photoemission spectroscopy was conducted by generating photoelectrons with the $\mathrm{Mg}-K_{\alpha}$ line $(1253.6 \mathrm{eV})$ from a PHI Model 05-548 dual-anode x-ray source, and measuring the electron energy with a large hemispherical electron energy analyzer (PHI Model 10-360 precision energy analyzer). The angle between the $\mathrm{x}$-ray source and the electron energy analyzer was fixed at $60^{\circ}$. The kinetic energies of the photoelectrons from carbon $1 s$ and fluorine $1 s$ core levels are about 963 and $563 \mathrm{eV}$, respectively, for excitation by the $\mathrm{Mg}-K_{\alpha}$ line $(1253.6 \mathrm{eV})$. The cross-section ratio of the $\mathrm{F} 1 \mathrm{~s}$ core level to the $\mathrm{C} 1 \mathrm{~s}$ core level is about 4.26 for excitation by the $\mathrm{Mg}-K_{\alpha}$ line. $^{25}$

The ARIPES spectra were obtained by using variable energy electrons, from a source based on the Erdman-Zipf design. ${ }^{26}$ For the ARIPES studies, the UV Geiger-Müller detector was employed with a mixture of iodine gas and helium gas at a pressure of 12 Torr. The iodine gas acts as a high pass filter, while a $\mathrm{CaF}_{2}$ window was used as a low-pass filter. ${ }^{27}$ In this scheme, $9.8-\mathrm{eV}$ photons were detected with the Geiger-Müller tube with a $\sim 400-\mathrm{meV}$ bandwidth. The overall energy resolution in inverse photoemission was $\sim 450 \mathrm{meV}$. The conduction-band spectra were taken by changing the kinetic energy of the incidence electrons from 5 to $19 \mathrm{eV}$. The wave-vector dependence was obtained by changing the electron incidence angle from $0^{\circ}$ (normal to the surface) to $55^{\circ}$ off-normal.

In the LEED measurements, angle-resolved core level photoemission and angle-resolved inverse photoemission studies, the 5-ML films of P(VDF-TrFE) were thin enough to conduct charge to the substrate at low-beam currents, thus avoiding significant surface charging that would affect the LEED or various electron spectroscopies. The Fermi level for photoemission, inverse photoemission, and core-level photoemission was established using a gold reference in contact with the sample substrate. The temperature was determined using a nickel-chromium vs nickel-aluminum $\left(T_{1}-T_{2}\right)$ thermocouple with an accuracy $\pm 3{ }^{\circ} \mathrm{C}$. The films were prepared by gentle annealing to $150{ }^{\circ} \mathrm{C}$ in vacuo, and surface composition was characterized with core-level x-ray photoemission spectroscopy.

The scanning tunneling microscope (STM) images were obtained on 2-ML films of P(VDF-TrFE) with sufficient tunneling current to obtain images with atomic resolution on a Digital Instruments Nanoscope III STM. X-ray-diffraction measurements were done using the $\mathrm{Cu}-K_{\alpha}$ line $(\lambda$ $\sim 1.54 \AA$ ) of several theta- 2 theta diffractometers.

Dielectric measurements were made at $1 \mathrm{kHz}$ with an HP 4192 impedance analyzer. A Keithly 498 current amplifier was used for conductance measurement at $0.1-\mathrm{V}$ bias. The pyroelectric measurements were conducted by measuring the ac sample current due to heating from an Ar-ion laser modulated at $1 \mathrm{kHz} .^{7,8}$

\section{LAYER CRYSTAL STRUCTURE AND THE BULK STRUCTURAL TRANSITION}

Studies of the bulk layer crystal structure were made for two film thickness. The $19^{\circ} \pm 1^{\circ}(2 \theta)$ x-ray-diffraction reflections were studied from 5- and 150-ML samples with

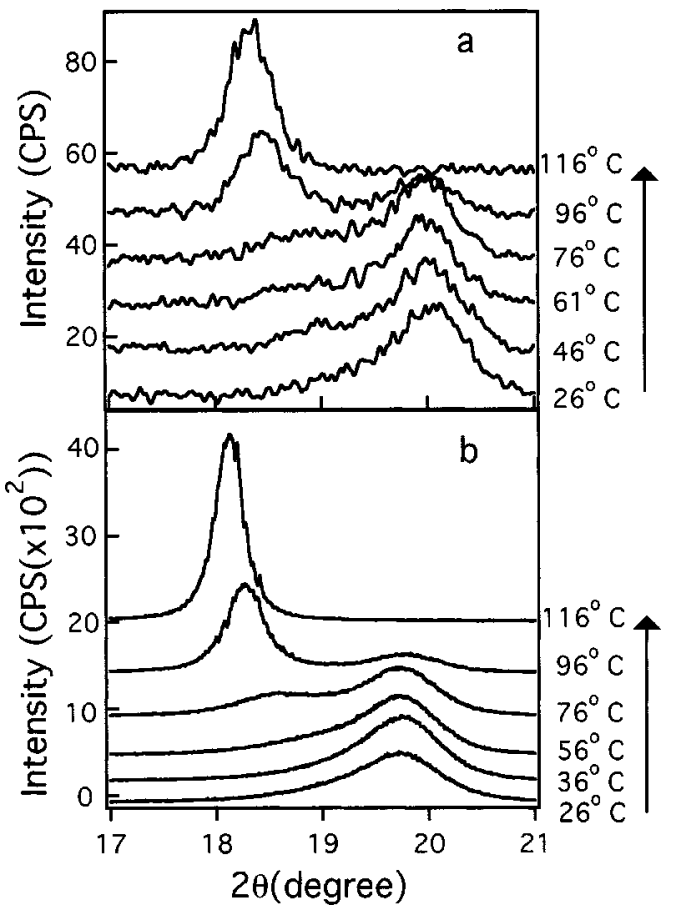

FIG. 1. X-ray-diffraction data of (a) a 5-ML and (b) a 150-ML $\mathrm{P}(\mathrm{VDF}-\mathrm{TrFE})$ film with increasing temperature.

increasing temperature, as shown in Fig. 1. These diffraction peaks are generally accepted to be an indication of the interlayer spacing along the polar (010) axis (normal to the film plane), ${ }^{12-15}$ and are consistent with x-ray-diffraction studies of $\mathrm{P}(\mathrm{VDF}-\mathrm{TrFE})$ fabricated by a variety of different techniques. ${ }^{13-15}$

While qualitatively similar to the published literature $^{12-15,22}$ and the results from thicker crystalline $\mathrm{P}(\mathrm{VDF}-\mathrm{TrFE})$ films [Fig. 1(b)], the $19^{\circ} \pm 1^{\circ}(2 \theta)(010)$ peak of the 5-ML films occurs at a slightly different $2 \theta$ angle, corresponding to a smaller lattice spacing between layers, $4.43 \AA$ as compared to a spacing of $4.48 \AA$ for the thicker 150-ML Langmuir-Blodgett films at room temperature.

There is a strong annealing effect in these $\mathrm{P}(\mathrm{VDF}-\mathrm{TrFE})$ Langmuir-Blodgett films. Annealing the 5- and 150-ML samples for an hour at $120^{\circ} \mathrm{C}$ results in almost a factor of 3 increase in the room-temperature Bragg diffraction peak height. We believe the annealing results in a diminution of void volume and an increase in domain size, so that the structural coherence length perpendicular to the film is effectively increased. ${ }^{14,28}$ Once adequately annealed, we observed no further changes to the film properties, apart from those changes associated with thermal expansion and with the reversible ferroelectric-paraelectric bulk phase transition.

Figure 1 shows the temperature dependence of the (010) Bragg peak for both the 5- and relatively thick 150-ML $\mathrm{P}(\mathrm{VDF}-\mathrm{TrFE})$ films across the $80^{\circ} \mathrm{C}$ bulk ferroelectric transition. ${ }^{7,8,12-15}$ At low temperatures (below $40^{\circ} \mathrm{C}$ ) and at high temperatures (above $100{ }^{\circ} \mathrm{C}$ ), only one phase is present, either the low-temperature ferroelectric phase $\left(2 \theta \approx 19.5^{\circ}\right.$ for the thicker $150-\mathrm{ML}$ films and $2 \theta \approx 20^{\circ}$ for the thinner 5 -ML films) or the high-temperature paraelectric phase ( $2 \theta$ $\approx 18.1^{\circ}$ for the thicker 150 -ML films and $2 \theta \approx 18.4^{\circ}$ for the thinner 5-ML films). At temperatures between 40 and 


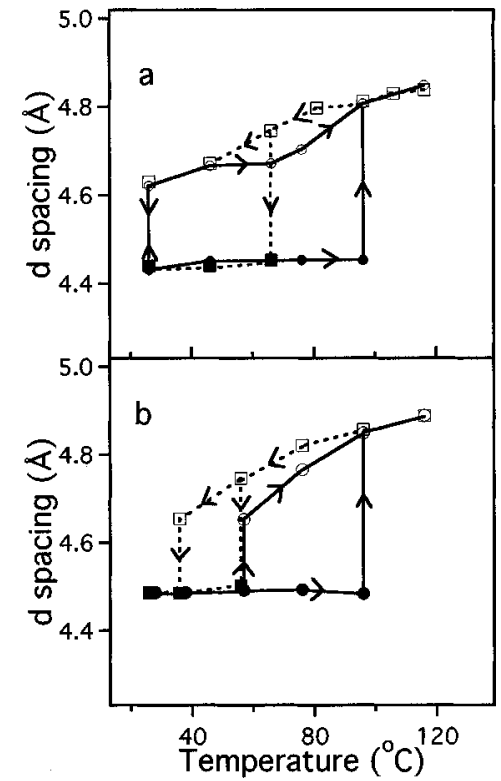

FIG. 2. Change of the layer spacing along the $\langle 010\rangle$ direction with increasing temperature for $d_{1}$ (ferroelectric phase, $\mathbf{O}$ ) and $d_{2}$ (paraelectric phase, $\bigcirc$ ), and with decreasing temperature for $d_{1}$ (ferroelectric phase, $\mathbf{\square}$ ) and $d_{2}$ (paraelectric phase, $\square$ ) for (a) a 5-ML and (b) a 150-ML film.

$100{ }^{\circ} \mathrm{C}$, there is a mixture of the two phases present, with one growing at the expense of the other. This can be clearly seen in Fig. 1, which shows the changes in the X-ray diffraction with increasing temperature. This is characteristic of the structural change in the $\mathrm{P}(\mathrm{VDF}-\mathrm{TrFE})$ bulk that accompanies the dielectric phase transition. ${ }^{12-14}$ The bulk paraelectric interlayer $d$ spacing, $\left(d_{2}=4.88 \AA\right.$ for $150-$ ML films and $d_{2}$ $=4.83 \AA$ for 5 -ML films), is larger than that in the bulk ferroelectric phase $\left(d_{1}=4.48 \AA\right.$ for $150-\mathrm{ML}$ films and $d_{1}$ $=4.43 \AA$ for 5 -ML films), as is now accepted. ${ }^{12-14}$ The general explanation is that the buckled $T G T \bar{G}$ chain combination of the paraelectric phase is "thicker" than the more compact linear all-trans conformation of the ferroelectric phase. ${ }^{14,29,30}$ The region of coexistence of the two phases is highly dependent on the rate of warming and cooling ${ }^{14}$ as would be expected for a first-order phase transition.

The temperature dependence of the lattice constants, calculated from the x-ray-diffraction peaks in Fig. 1, is shown in Fig. 2. Once again the large hysteresis in the region of coexistence is a consequence of the first-order phase transition. An interesting point is that the lower curve is almost completely flat with temperature, indicating that the interlayer spacing of the ferroelectric phase is constant as a function of temperature, particularly for the thicker films. For the bulk paraelectric phase, denoted by the upper part of the loop, there is a strong temperature dependence. This differs from the results on spin-cast films, ${ }^{12-14}$ which show a small temperature dependence in both phases. It is possible that differences in microstructure and crystallite sizes in the spincast films, as opposed to our Langmuir-Blodgett fabricated films, is the origin of these differences. The structural hysteresis of Fig. 2 is also observed in the very thick spun film as thick as $0.5 \mathrm{~mm} .^{12-14}$

These $\mathrm{x}$-ray-diffraction results establish that the bulk layer spacing for both thick (150 ML) and thin (5 ML)

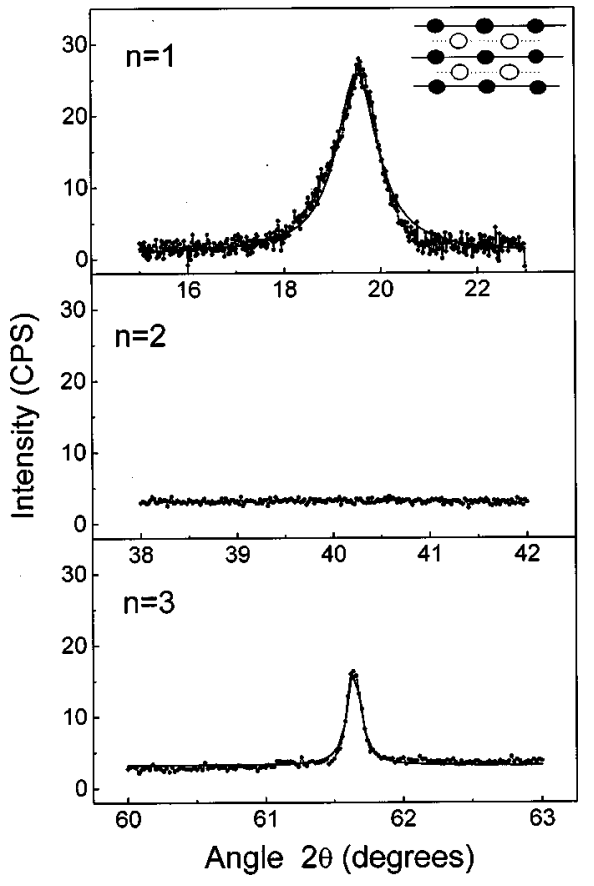

FIG. 3. Rotating anode x-ray scattering data for a 100-ML film of $\mathrm{P}(\mathrm{VDF}-\mathrm{TrFE})$. Note the presence of the odd order diffraction peaks and the absence of the even orders. The slit aperture was increased for $n=2$ and 3 over $n=1$ to increase the count rates for the high-order reflections. The inset (top right) is a schematic of the chain packing, end on to the chains (the chains run into the page). The layer-to-layer spacing (the horizontal rows of filled symbols) is $4.5 \AA$.

Langmuir-Blodgett films of $\mathrm{P}(\mathrm{VDF}-\mathrm{TrFE})$ are qualitatively similar. The $\mathrm{x}$-ray-diffraction data show a clear broadening of the peak in the low-temperature phase. Possible explanations include the existence of a mixture of $(010)+(100)$ domains or a change in the coherence length. The (010) + (100) spacings are close, $4.48 \AA$ as compared to $4.29 \AA$, but not identical. The peak widths tend not to support the first explanation, as the two domains would be manifest as two separate peaks. A calculation of the coherence length using Debye-Scherrer formula $\left(L_{c}=0.9 \lambda / \Delta 2 \theta \cos \theta_{B}\right)$ gives a coherence length of $\sim 300 \AA$ in the high-temperature phase and $\sim 100 \AA$ in the low temperature phase in the 150 -ML films.

The coherence length is large enough for us to observe higher-order diffraction spots for the 100-ML film, as seen in Fig. 3. In addition to the fundamental peak at $19.8^{\circ}$ (corresponding, as noted to approximately the 4.5 - $\AA$ layer spacing) higher-order peaks are also present. The absence of the second-order peak (and presumably all even-numbered peaks) can be explained by a super-periodicity due to a shift of the longitudinal chain spacing in one plane to the next. This is consistent with a shift of $\frac{1}{2}$ of a $-\mathrm{CH}_{2}-\mathrm{CF}_{2}$ monomer spacing (or $\frac{1}{2}$ of $2.56 \AA$ ) or the $\frac{1}{4}$ unit-cell phase slip observed in the surface [Fig. 4(a)]. Consider the unit cell, as shown in the inset of Fig. 3, in which the polymer chains are perpendicular to the plane of the paper. The chains depicted in filled black are displaced half a monomer unit along the chain direction with respect to the open symbols. This slippage results in a destructive interference between the $\mathrm{x}$-rays scat- 

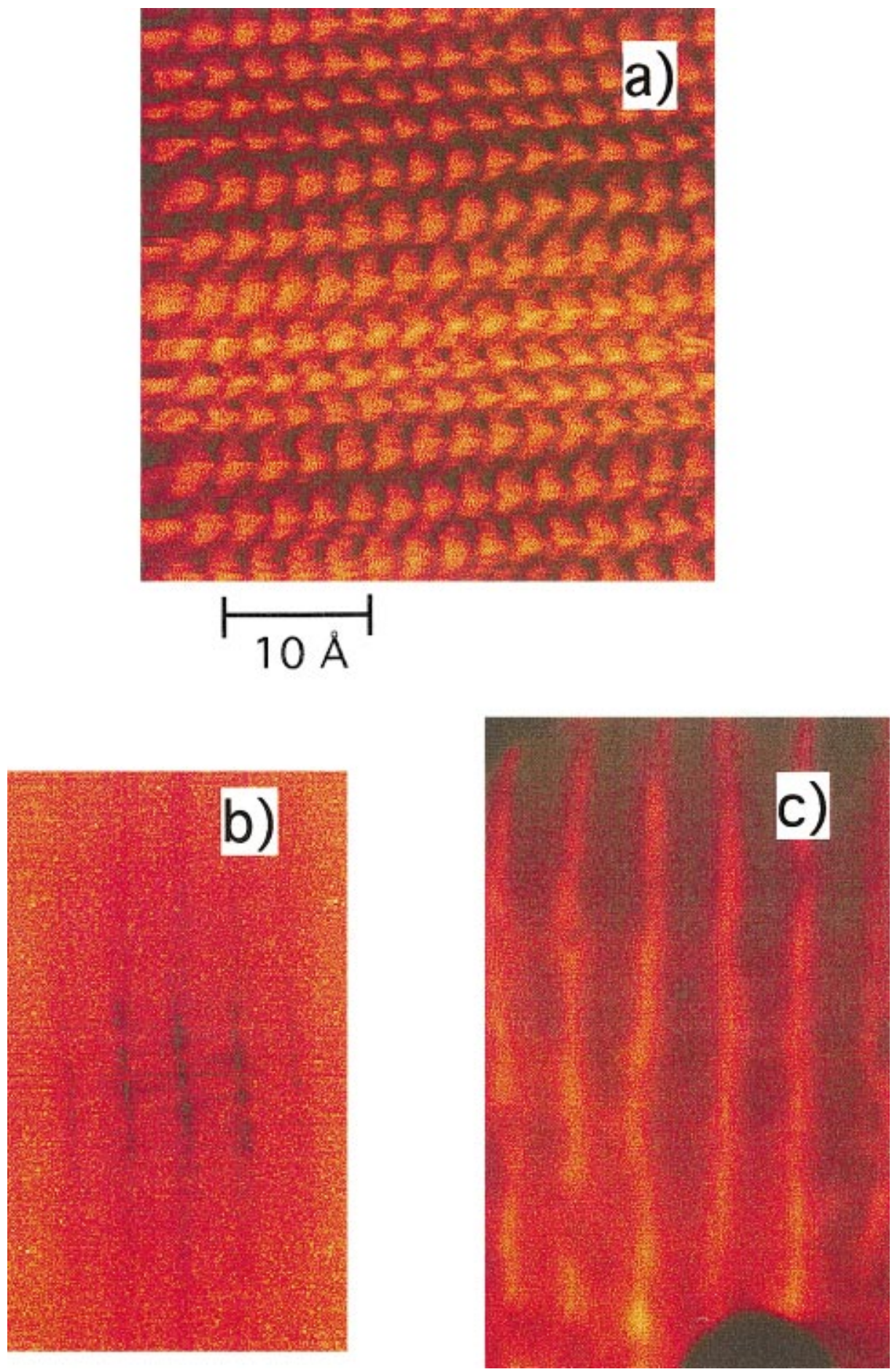

FIG. 4. (Color) The surface structure of crystalline P(VDF-TrFE) Langmuir-Blodgett films. (a) A scanning tunneling microscope image $\left(40 \times 40 \AA^{2}\right)$, recorded at $295 \mathrm{~K}$, of a 2-ML P(VDF-TrFE) film on a graphite substrate. (b) The Fourier transform of the STM image (a). (c) The low-energy electron-diffraction image of a 5-ML P(VDF-TrFE) film on silicon taken at $200 \mathrm{~K}$.

tered off the filled black and open symbol layers; hence only the odd harmonics remain.

\section{SURFACE CRYSTAL STRUCTURE}

Surface crystal structure was studied using multiple techniques. The (STM) image, recorded at room temperature, for a 2-ML P(VDF-TrFE) film on a cleaved graphite substrate, is shown in Fig. 4(a). The in-plane spacing between the polymer chains is distributed from 3.5 to $4.3 \AA$, which is some- what smaller than is generally observed in the bulk crystal structure, ${ }^{12-19}$ because intervening layers in the bulk keep the chains further apart. The STM image suggests that there exists a surface reconstruction or relaxation of the sublayer of polymer chains toward the surface, as schematically shown in Fig. 5(a).

Along the chain, as determined from the STM image, the spacing between monomers is about $2.5 \pm 0.1 \AA$, in good agreement with the bulk crystal structure. ${ }^{12-19}$ The observed chain structure appears to correspond to the all-trans surface 


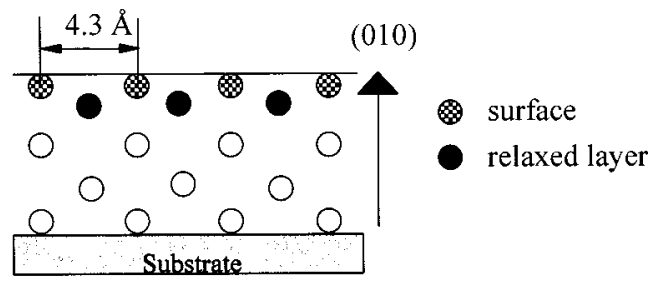

a)

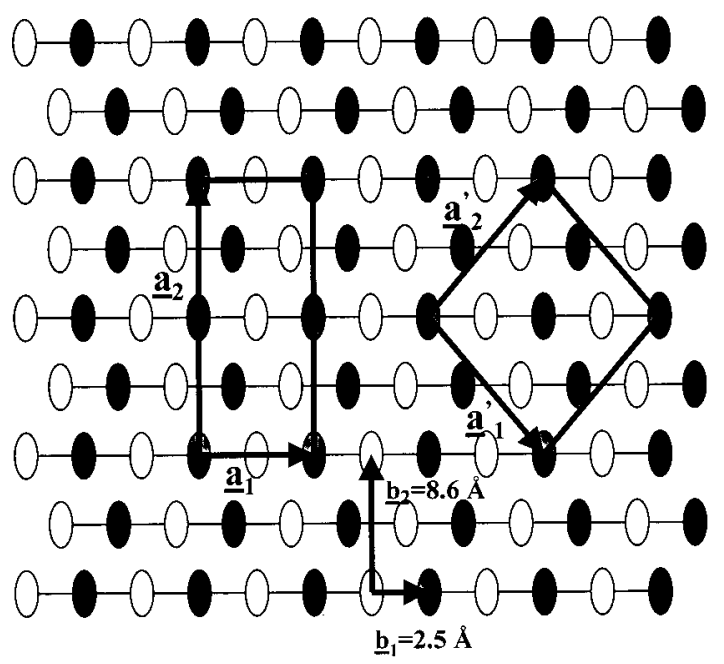

b)

FIG. 5. Schematic diagram of the surface crystal structure in real space based on the scanning tunneling microscopy and the low-energy electron-diffraction studies in an all-trans conformation of $\mathrm{P}(\mathrm{VDF}-\mathrm{TrFE})$. A relaxation of subsurface layer occurs (a) based on STM while the surface layer (b) could be described by either a rectangular primitive cell with eight $\mathrm{CH}_{2}-\mathrm{CF}_{2}$ monomers as a basis or an oblique primitive cell with eight $\mathrm{CH}_{2}-\mathrm{CF}_{2}$ monomers as a basis. The lines in (b) indicate the chain direction, and a possible, but not unique packing of the $\mathrm{CH}_{2}-\mathrm{CF}_{2}$ monomers with respect to the $\pm 7^{\circ}$ tilt (see text) is indicated by shading.

ferroelectric phase of the random copolymer of vinylidene fluoride $\quad(70 \%)$ with trifluoroethylene $(30 \%)$ $\left[\left(-\mathrm{CH}_{2}-\mathrm{CF}_{2}\right)_{0.7}-\left(\mathrm{CH}_{2}-\mathrm{CHF}-\right)_{0.3}\right]$. The relative longitudinal position between the $\mathrm{CH}_{2}-\mathrm{CF}_{2}$ dimer pairs in neighboring chains inplane appears shifted by $\frac{1}{2}$ dimer spacing. Since the unit cell consists of two dimers, this corresponds to a $\frac{1}{4}$ unitcell phase slip. Because of the surface relaxation, the slip from chain to chain at the surface is not expected to be present in the bulk layers, but rather is manifest as a longitudinal slip from one plane to the next. (This is the origin of the x-ray data discussed above.)

The LEED images [Fig. 4(c)] of 5-ML films of P(VDFTrFE, 70:30) on $\mathrm{Si}(111)$ taken at $200 \mathrm{~K}$ are, as expected, qualitatively similar to the Fourier transformation [Fig. 4(b)] of the real-space surface crystal structure of $\mathrm{P}(\mathrm{VDF}-\mathrm{TrFE})$ film taken by a scanning tunneling microscope [Fig. 4(a)]. The disorder of interchain spacing (perpendicular to chain) in real space is represented as stripes in momentum $(k)$ space in both Figs. 4(a) and 4(b). Because of this disorder, the scanning tunneling microscopy and low-energy electrondiffraction studies are consistent with surface primitive cells that are either a rectangular or a pseudohexagonal structure. These two possible surface crystal structures are consistent within the experimental error with the Brillouin-zone critical points determined from the experimental conduction band mapping, as listed in Table I. As yet, neither potential structure can be definitely eliminated. In spite of problems with disorder, the LEED data reflect a periodicity of the interchain spacing (or a modulation in the stripes) not apparent in the STM image, as summarized in Table I.

In the low-energy electron diffraction, the unit-cell dimension is actually two $-\mathrm{CH}_{2}-\mathrm{CF}_{2}$ monomer units long (a dimer). The low-energy electron diffraction is sensitive to the orientation of the fluorine orbitals which may alternate between $\pm 7^{\circ}$ with respect to the chain axis, and $x$-ray scattering is insensitive to this variation within the layer spacing as is scanning tunneling microscopy. The two possible realspace surface lattice structures are shown in Fig. 5(b). The potential rectangular surface structure packing scheme contains eight monomers. This structure is postulated based on the scanning tunneling microscopy study and low-energy electron-diffraction study (Fig. 4), with lattice constants $\mathbf{a}_{1}$ and $\mathbf{a}_{2}$ as indicated in Fig. 5(b). The choice of the basis vector along $\mathbf{a}_{1}$, as twice the distance between monomer units (rather than the distance between monomer units), is dictated by the experimental band-structure mapping which will be discussed in a later section. The primitive lattice constant along the $\mathbf{a}_{1}$ direction from the band structure is $\sim 4.8$

TABLE I. Comparison of the modeled surface Brillouin zones at the surface ferroelectric phase based on scanning tunneling microscopy and a low-energy electron, diffraction study with a conduction-band structure obtained from angle resolved inverse photoemission. All units are in $\AA^{-1}$. Along the $\mathbf{a}_{2}^{*}$ direction of the Brillouin zone, LEED must be considered the most definitive value.

\begin{tabular}{|c|c|c|c|c|}
\hline Structure & $\begin{array}{l}\text { Critical points/ } \\
\text { directions }\end{array}$ & $\begin{array}{c}\mathbf{a}_{1}^{*} \\
\left(\AA^{-1}\right)\end{array}$ & $\begin{array}{c}\mathbf{a}_{2}^{*} \\
\left(\AA^{-1}\right)\end{array}$ & $\begin{array}{c}\mathbf{a}_{3}^{*} \\
\left(\AA^{-1}\right)\end{array}$ \\
\hline STM & $\begin{array}{l}\text { Zone center to } \\
\text { zone center }\end{array}$ & $1.26 \pm 0.04$ & $0.73 \pm 0.1$ & $1.26 \pm 0.1$ \\
\hline LEED & $\begin{array}{l}\text { Zone center to } \\
\text { zone center }\end{array}$ & $1.26 \pm 0.04$ & $0.37 \pm 0.05$ & $1.46 \pm 0.05$ \\
\hline \multirow{2}{*}{$\begin{array}{l}\text { Experimental } \\
\text { band structure }\end{array}$} & Zone edge & $0.65 \pm 0.04$ & ------------- & $0.75 \pm 0.05$ \\
\hline & $\begin{array}{l}\text { Zone center to } \\
\text { zone center }\end{array}$ & $1.30 \pm 0.04$ & ------------- & $1.26 \pm 0.05$ \\
\hline
\end{tabular}




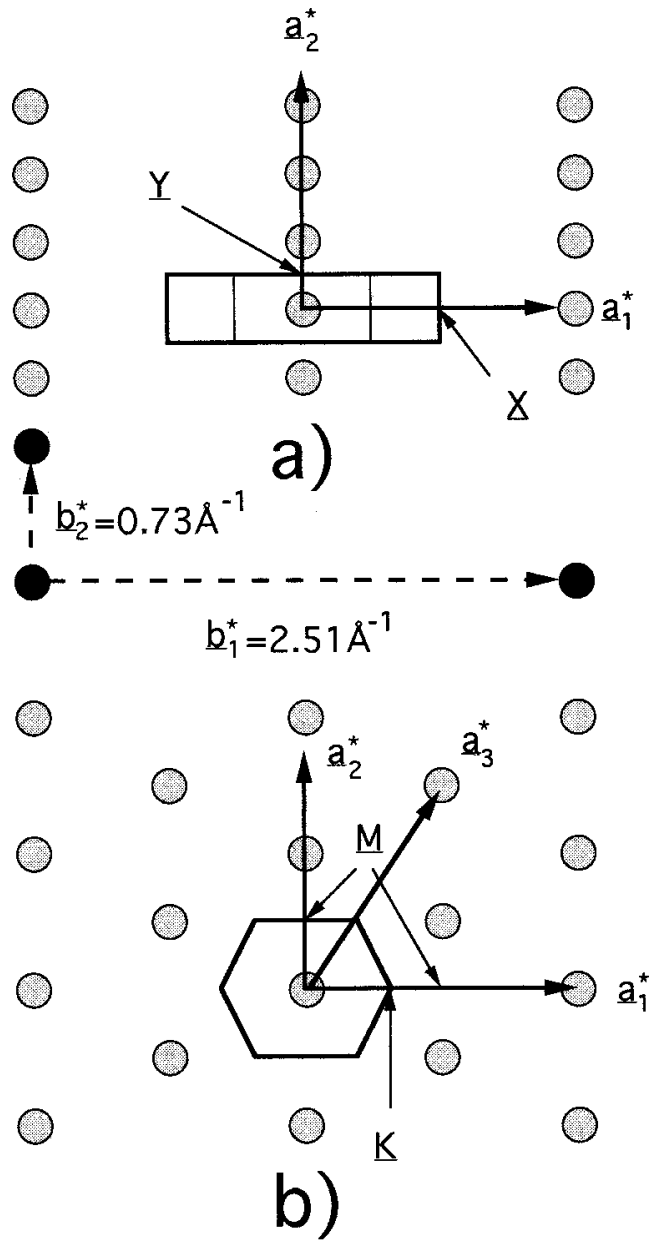

FIG. 6. Schematic diagram of the surface crystal structure in $k$-momentum space of the all-trans conformation of $\mathrm{P}(\mathrm{VDF}-\mathrm{TrFE})$ of (a) a rectangular primitive cell with eight monomers per unit cell, and (b) of an oblique primitive cell with eight monomers per unit cell. The polygons show the first Brillouin zone at the ferroelectric phase in each case.

$\AA$ (in the surface ferroelectric phase); hence the two choices of unit cell.

The real-space primitive translation vectors of the surface can be expressed in terms of the projected real-space translation vectors, $\mathbf{b}_{1}=2.5 \AA$ and $\mathbf{b}_{2}=8.6 \AA$ (Ref. 22) onto the (010) plane ${ }^{12-19}$ by the matrix $\mathfrak{R}(A=\mathfrak{R} B)$ (Ref. 31) for the possible rectangular surface structure as

$$
\left(\begin{array}{l}
\mathbf{a}_{1} \\
\mathbf{a}_{2}
\end{array}\right)=\left(\begin{array}{ll}
2 & 0 \\
0 & 2
\end{array}\right)\left(\begin{array}{l}
\mathbf{b}_{1} \\
\mathbf{b}_{2}
\end{array}\right) .
$$

The surface reciprocal-space primitive translation vectors are obtained using the matrix relationship $A^{*}=\tilde{\mathfrak{R}}^{-1} B^{*}$, where the projected bulk primitive reciprocal-lattice vectors, in $k$ space, are $\mathbf{b}_{1}^{*}=2.51 \AA^{-1}$ and $\mathbf{b}_{2}^{*}=0.73 \AA^{-1}$ and $\tilde{\Re}^{-1}$ is the inverse transpose of $\mathfrak{R}$ (Ref. 29) as

$$
\left(\begin{array}{l}
\mathbf{a}_{1}^{*} \\
\mathbf{a}_{2}^{*}
\end{array}\right)=\left(\begin{array}{cc}
\frac{1}{2} & 0 \\
0 & \frac{1}{2}
\end{array}\right)\left(\begin{array}{l}
\mathbf{b}_{1}^{*} \\
\mathbf{b}_{2}^{*}
\end{array}\right) .
$$

This postulated surface crystal structure, in $k$ space, is as shown in Fig. 6(a).
Another possible surface lattice structure resembles a distorted hexagon. This structure is suggested by the Fourier transform [Fig. 4(b)] of the real surface crystal structure, and also cannot be excluded based upon the available data for the surface primitive cell of the copolymer films of P(VDFTrFE). This pseudohexagonal surface lattice structure in real space can be described using the matrix relation

$$
\left(\begin{array}{l}
\mathbf{a}_{1} \\
\mathbf{a}_{2}
\end{array}\right)=\left(\begin{array}{cc}
2 & -1 \\
2 & 1
\end{array}\right)\left(\begin{array}{l}
\mathbf{b}_{1} \\
\mathbf{b}_{2}
\end{array}\right)
$$

The reduced reciprocal-lattice structure from Eq. (3) is shown in Fig. 6(b). The semi-hexagonal structure in $k$ space is represented as

$$
\left(\begin{array}{l}
\mathbf{a}_{1}^{*} \\
\mathbf{a}_{2}^{*}
\end{array}\right)=\left(\begin{array}{cc}
\frac{1}{4} & -\frac{1}{2} \\
\frac{1}{4} & \frac{1}{2}
\end{array}\right)\left(\begin{array}{l}
\mathbf{b}_{1}^{*} \\
\mathbf{b}_{2}^{*}
\end{array}\right) .
$$

Even though these two possible surface lattice structures, based on scanning tunneling microscopy and low-energy electron-diffraction studies, have two different effective Brillouin-zone shapes and sizes, the critical points are consistent with the conduction-band-structure studies. The conduction-band structure agrees somewhat better with the pseudohexagonal surface structure rather than the rectangular structure, but, given our experimental uncertainties, this is not compelling. Conduction-band mappings ${ }^{9}$ are consistent with this surface structure.

Angle-resolved inverse photoemission spectra were taken along three high-symmetry directions $\mathbf{a}_{1}^{*}, \mathbf{a}_{2}^{*}$, and $\mathbf{a}_{3}^{*}$, indicated in Fig. 6(b). With some directional uncertainty, $\pm 5^{\circ}$, this band structure for 5-ML P(VDF-TrFE) films grown on $\mathrm{Si}(111)$ substrates, as detailed elsewhere, ${ }^{9}$ does provide some insight into the surface structure. The conduction-band dispersion of the lowest unoccupied molecular-orbital states along $\mathbf{a}_{1}^{*}, \mathbf{a}_{2}^{*}$, and $\mathbf{a}_{3}^{*}$ directions (adapted from Ref. 9) are summarized as a function of $\mathbf{k}_{\|}$, the wave vector parallel to the plane of the surface, in Fig. 7. Figure 7(a) shows the dispersion of the first unoccupied (conduction band) state along the polymer chain $\left(\mathbf{a}_{1}^{*}\right)$ below $(200 \mathrm{~K}$, circles) and above $(320 \mathrm{~K}$, squares) the surface ferroelectric transition. The amplitude of the conduction band dispersion is about 1.5 $\mathrm{eV}$ in both phases. The period of conduction-band dispersion above the surface ferroelectric phase transition temperature (at $320 \mathrm{~K}$ ) is clearly shorter (in $k$ space) than below the surface ferroelectric phase transition temperature (at $200 \mathrm{~K}$ ). Along the $\mathbf{a}_{1}^{*}$ high-symmetry direction of the surface Brillouin zone (parallel to the polymer chains), the distance from Brillouin-zone center to zone center decreases by nearly half from $1.30 \pm 0.04$ to $0.73 \pm 0.05 \AA^{-1}$ across the surface ferroelectric transition. Figure $7(\mathrm{c})$ shows the conduction-band dispersion along $\mathbf{a}_{3}^{*}$ (see Fig. 6) for the lowest unoccupied molecular-orbital state. The dispersion along $\mathbf{a}_{3}^{*}$ is qualitatively similar to the band dispersion along $\mathbf{a}_{1}^{*}$ [parallel to the polymer chain; see Fig. 7(a)], showing the same halving of the Brillouin zone (doubling of the real-space period) from $1.26 \pm 0.05$ to $0.80 \pm 0.05 \AA^{-1}$ across the surface phase transition. The amplitude of the band dispersion along the $\mathbf{a}_{3}^{*}$ direction (about $1.3 \mathrm{eV}$ ) is again large, as is the case along the direction of the polymer chain, $\mathbf{a}_{1}^{*}(1.5 \mathrm{eV})$. 


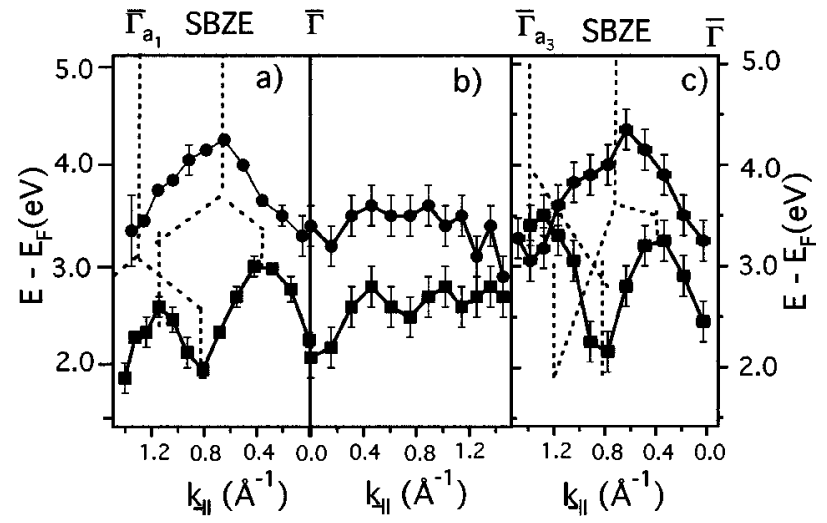

FIG. 7. The band dispersion of the lowest unoccupied molecular-orbital (LUMO) states in the surface ferroelectric phase $(\boldsymbol{O})$ at $200 \mathrm{~K}$, and in the surface paraelectric phase (ם) at $320 \mathrm{~K}$ (a) along the $\mathbf{a}_{1}^{*}$ direction of the Brillouin zone (along the chains), (b) along the $\mathbf{a}_{2}^{*}$ direction of the Brillouin zone (perpendicular to the chains), and (c) along the $\mathbf{a}_{3}^{*}$ direction of the Brillouin zone [see Fig. 6(b) for the labelling of the Brillouin-zone symmetry directions]. The wave vector of the Brillouin-zone edge is indicated by SBZE and the Brillouin-zone center by $\bar{\Gamma}$. The dashed lines indicate the Brillouin zone centers and zone edges in the ferroelectric and paraelectric surface phases. Adapted from Ref. 9.

The magnitude of conduction-band dispersion perpendicular to the chain $\left(\mathbf{a}_{2}^{*}\right)$ is relatively small, $\leqslant 200 \mathrm{meV}$, and the Brillouin-zone edge cannot be determined, as shown in Fig. 7(b). From low-energy electron diffraction, we anticipate that there is a surface Brillouin-zone edge along $\mathbf{a}_{2}^{*}$ at about $0.19 \pm 0.05 \AA^{-1}$. This is based upon the LEED intensity modulation along this direction. The small conductionband dispersion amplitude along $\mathbf{a}_{2}^{*}$ is an indication of the weak interaction (hybridization of molecular orbitals) between polymer chains and the disorder between chains, and may also be limited by finite experimental resolution.

The surface Brillouin-zone edge ( $\bar{M}$ or $\bar{X}$ in Fig. 6) along $\mathbf{a}_{1}^{*}$ and the surface Brillouin-zone edge along $\mathbf{a}_{3}^{*}$ in the alltrans conformation, are at about $0.65 \pm 0.04$ and 0.42 $\pm 0.05 \AA^{-1}$ respectively. The $k_{\|}$wave-vector scanning along $\mathbf{a}_{3}^{*}$ could cross another zone edge $(\bar{X})$ which is placed at $0.71 \pm 0.05 \AA^{-1}$. The Brillouin-zone edge ( $\bar{M}$ or $\bar{Y}$ in Fig. 6) along $\mathbf{a}_{2}^{*}$ is about $0.19 \pm 0.05 \AA^{-1}$, and there is no apparent change of the Brillouin-zone size along $\mathbf{a}_{2}^{*}$ across the surface phase transition. As shown in Table I, the surface Brillouinzone critical points, derived from three independent measurements:, scanning tunneling microscopy, low-energy electron diffraction, and angle-resolved inverse photoemission spectroscopy, are consistent. ${ }^{9}$

\section{SURFACE COMPOSITION CHANGES}

The observed large amplitude and period doubling of the conduction-band dispersion along the polymer chain of the copolymer films of $\mathrm{P}(\mathrm{VDF}-\mathrm{TrFE})$ is similar to theoretical calculations of the conduction-band structure as a function of the conformational structural change along the polymer chain of polyethylene and polytetrafluoroethylene. ${ }^{32-35}$ The conduction-band structure suggests that the Brillouin-zone size, along $\mathbf{a}_{1}^{*}$ (along the polymer chain), is almost two times

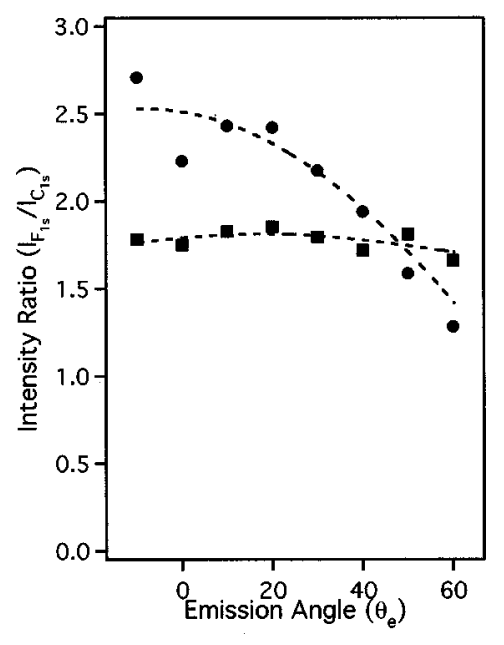

FIG. 8. The intensity ratio of the fluorine $1 s$ core-level spectra intensity to carbon $1 s$ core-level spectra intensity as a function of the photoelectron emission angle $(\mathbf{O})$ at $200 \mathrm{~K}$ and $(\boldsymbol{\square}) 320 \mathrm{~K}$.

larger in the low-temperature surface ferroelectric phase than the distance from the Brillouin-zone center to the Brillouinzone center of the high temperature surface phase [Fig. 7(a)]. The Brillouin-zone size doubling is possibly the result of the conformational structure change from an all-trans (TT) configuration to an alternating gauche-trans $(T G T \bar{G})$ configuration. ${ }^{14,29,30}$ Such a change implies a significant periodicity change along the polymer chain from $5 \AA$, at low temperatures, to $9.2 \AA$ or nearly double, at higher temperatures. The $T G T \bar{G}$ chains are slightly shorter overall than the $T T$ chains due to the longitudinal contraction of the $G$ and $\bar{G}$ bonds relative to the $T$ bonds. This too is consistent with our data.

The conformational changes across the surface ferroelectric phase transition, by necessity, change the surface composition as the gauche bonds move atoms to and from the surface. The surface composition changes are revealed by angle-resolved core-level photoemission spectroscopy across the surface phase transition. The core-level spectra are surface sensitive and therefore emission angle dependent, because the mean free path of the photoelectrons is small, usually less than $10 \AA$, and roughly proportional to the square root of the emerging electron kinetic energy within the energy range of $100 \mathrm{eV}$ to $6 \mathrm{KeV} .{ }^{36}$ The escape probability of the photoelectron emission decreases, with increasing angle $\theta_{e}$ of emission, as $\cos \left(\theta_{e}\right)$. Therefore, the intensity and angle dependence of core-level photoemission serves as a useful measure of the relative position of the source atoms. ${ }^{37,38}$

This is illustrated in Fig. 8, which shows the angular distribution of the ratio of the fluorine $1 s$ core level (binding energy $\sim 686 \mathrm{eV}$, kinetic energy $\sim 563 \mathrm{eV}$ ) photoelectron emission intensity to the carbon $1 s$ core level (binding energy $\sim 286 \mathrm{eV}$, kinetic energy $\sim 963 \mathrm{eV}$ ) photoelectron intensity both below $(200 \mathrm{~K})$ and above $(320 \mathrm{~K})$ the surface ferroelectric phase transition temperature. Since polymer conformation change does not cause a net displacement of carbon, the fluorine/carbon core-level photoelectron intensity ratio is a measure of the surface fluorine density.

Below the surface ferroelectric transition temperature, the core-level intensity ratio (circles in Fig. 8) falls off rapidly with increased emission angle, showing that the fluorine atoms are predominantly below the surface carbon atoms. The 


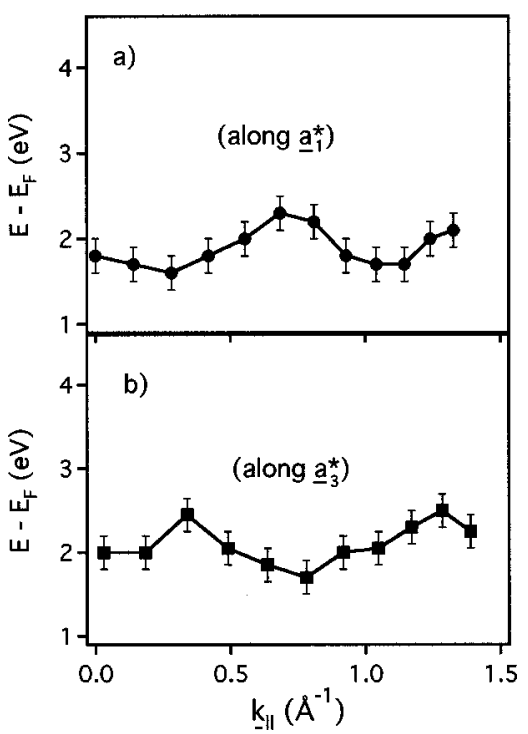

FIG. 9. The conduction-band structure above the bulk phase transition temperature at $90^{\circ} \mathrm{C}$ (a) along, $\mathbf{a}_{1}^{*}$ and (b) $\mathbf{a}_{3}^{*}$.

surface layer polarization is therefore directed outward, pointing from the buried fluorine to the hydrogen on top. In contrast, above the surface ferroelectric transition temperature, the intensity ratio is almost independent of emission angle indicating that as many of the fluorines are now above the surface as below or that fluorine and carbon are coplanar. Since the $T G T \bar{G}$ conformation of the high-temperature phase has an equal distribution of fluorine on both sides of the surface layer (considering the sublayer relaxation), the flat intensity ratio (Fig. 8, squares) is consistent with either normal or in-plane orientation of the carbon backbone.

The observation that the fluorine atoms are below the plane of the surface in the ferroelectric phase, even without applying a polarizing field, is a satisfying confirmation of previous observations that films thinner than 15 ML preferentially polarize with the hydrogens on top and the fluorine buried. ${ }^{7,24,34,39}$ Additional evidence for this dipole alignment change across the surface transition can be found from the work-function change, as noted elsewhere. ${ }^{8,9}$

\section{TEMPERATURE-DEPENDENT DISORDER}

Above the bulk phase transition temperature, the conduction-band dispersion at $90^{\circ} \mathrm{C}$ along $\mathbf{a}_{1}^{*}$ and $\mathbf{a}_{3}^{*}$ of the Brillouin zone (see Fig. 6) are shown in Fig. 9. The amplitude of the band dispersion in both directions is less than 500 $\mathrm{meV}$ and the Brillouin-zone periodicity is difficult to ascertain. The relatively small dispersion amplitude compared to the results at several lower temperatures (Fig. 7), and diminishing resolution of the Brillouin-zone dispersion periodicity are a good indication of disorder connected with structure (spatial or temporal) fluctuations as the ordered $T G T \bar{G}$ chain structures undergoes dynamic distortions to other conformations with increasing temperature. While the "bulk transition" may be manifest in films as thin as $2 \mathrm{ML}^{7}$ there is no compelling evidence of the $80^{\circ} \mathrm{C}$ bulk transition in inverse photoemission. This may be because angle-resolved inverse photoemission is a surface-sensitive spectroscopy, and the changes in band structure with ever increasing temperature
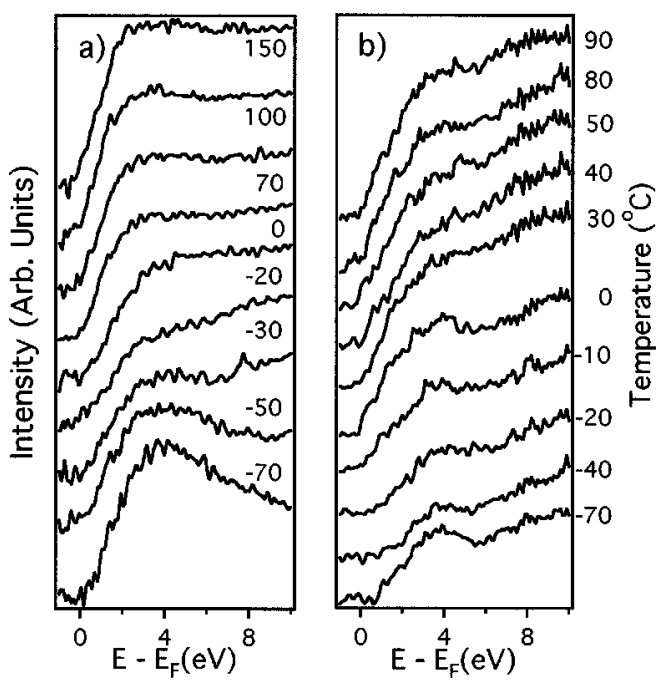

FIG. 10. The inverse photoemission spectra as a function of temperature taken (a) at the Brillouin-zone center $(\bar{\Gamma})$ and (b) at the surface Brillouin-zone edge $(X$ or $M)$.

above the surface ferroelectric transition cannot be directly associated with the bulk transition.

The conduction-band spectra, recorded at the zone center $\bar{\Gamma}$ and at the zone edge for the low-temperature surface ferroelectric phase along $\mathbf{a}_{1}^{*}$ (the polymer chain axis), are shown in Fig. 10. The shift of the density of states toward the Fermi level, as the temperature is increased, is seen at both points in reciprocal space. It is this shift in the density of states that is responsible for the change in metallicity shown earlier. ${ }^{8}$ These electronic structure changes (density of states near Fermi level in the unoccupied side and the position of the lowest unoccupied molecular orbital) are compared to the bulk properties as functions of temperature in Fig. 11. The density of states near the Fermi level at two different points in the Brillouin zone, at the zone center (opened circles) and at the zone edge (filled circles) show a significant change around the surface phase transition temperature $\left(\sim 20^{\circ} \mathrm{C}\right)$. The change of the density of states at the zone edge is more dramatic than at the zone center, as expected, from the shift of the lowest unoccupied molecular orbital states and the instability of the Brillouin-zone edge as shown in Fig. 7. Near to the surface phase transition $\left(\sim 20^{\circ} \mathrm{C}\right)$, the electronic structure (density of states near the Fermi level and shift of lowest unoccupied molecular-orbital states) dramatically changes but further significant changes in electronic structure, related to the bulk ferroelectric transition at $80^{\circ} \mathrm{C}$, are not observed. As noted elsewhere, ${ }^{1,8,9}$ we conclude that inverse photoemission is surface sensitive. Thus the conduction-band structure, as indicated in the preceding discussion, is representative of the surface, not the bulk.

There are, nonetheless, strong indications of both surface and bulk phase transitions of the 5-ML P(VDF-TrFE) films. Both surface $\left(\sim 20^{\circ} \mathrm{C}\right)$ and bulk $\left(\sim 80^{\circ} \mathrm{C}\right)$ phase transitions of 5-ML P(VDF-TrFE) films are observed in the dc conductivity as shown in Fig. 11(c). The pyroelectric response change of the spontaneous polarization due to temperature modulation shows, for 5-ML P(VDF-TrFE) films, both the surface and bulk phase transitions as shown in Fig. 11(c). The changes of the transport properties, dc conductivity, and 

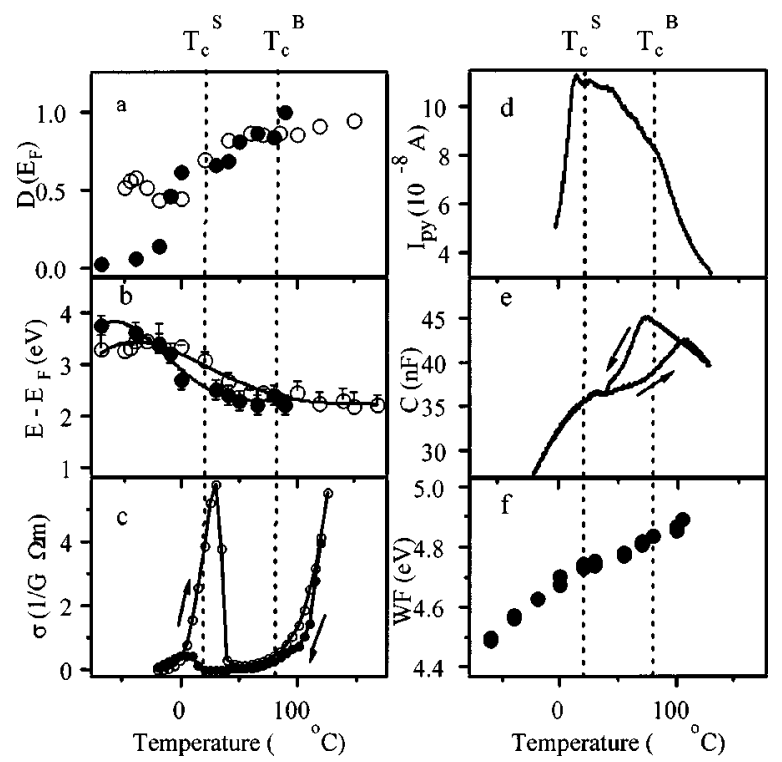

FIG. 11. Temperature dependence of several physical quantities showing one or both of the surface phase transitions. (a) The integrated density of states within a binding energy of $0.5 \mathrm{eV}$ above the Fermi level derived from inverse photoemission at the zone center (O) and at the zone edge $(\bigcirc)$, in 5-ML P(VDF-TrFE) films. (b) The binding-energy shift of the LUMO states above $E_{F}$. (c) The conductivity through a 5-ML film. (d) The pyroelectric current. (e) The capacitance. (f) The work function.

pyroelectric current across the surface phase transition are consistent with changes of the unoccupied electronic band structures [Figs. 11(a) and 11(b)]. Capacitance measurements of 5-ML P(VDF-TrFE) films [Fig. 11(e)] also show peaks at both the surface and bulk ferroelectric-paraelectric phase transitions. ${ }^{7,8}$ The surface phase transition is consistent with the slow change of the work function with respect to the sample temperature across the surface phase transition, as shown in Fig. 11(f).

Three bulk measurements-dc conductivity, pyroelectric current, and capacitance measurements - show that there is a low-temperature phase transition which is demonstrably the surface phase transition, as shown by the surface-sensitive spectroscopies (angle-resolved inverse photoemission, angleresolved core-level photoemission, and diode work-function measurements). The studies, using these surface-sensitive spectroscopies, indicate that the surface is increasingly disordered with increasing temperature.

\section{DISCUSSION}

The bulk crystal structure and the structure changes across the ferroelectric to paraelectric phase transition of the copolymer poly vinylidene fluoride-trifluoroethylene P(VDFTrFE, 70:30) films grown by the Langmuir-Blodgett method are qualitatively similar to films fabricated by the other methods, such as a spin casting or melt drawing. ${ }^{12-19}$ This is demonstrated in Figs. 1 and 2, though the crystal order in the Langmuir-Blodgett (LB)-grown films is significantly greater and a superperiodicity is evident, as seen in Fig. 3.

The bulk crystal structure of the P(VDF-TrFE) copolymers has been a subject of some controversy. ${ }^{40}$ Initial $\mathrm{X}$-ray studies suggested that in the ferroelectric phase the structure is identical to that of pure PVDF in the ferroelectric state, i.e., an orthorhombic symmetry. ${ }^{29}$ However, in most copolymer mixtures, there is a mixture of two crystalline phases that coexist- an orthorhombic structure (the so-called low temperature phase) and a distorted hexagonal structure (the cooled phase). ${ }^{41}$ The proportion of phases at a fixed temperature is dependent on both the method of cooling as well as the ratio between the two copolymers. These results are for bulk samples, while in our LB films, the growth conditions and the presence of a minimal amount of amorphous phase may lead to very different structures. In the LB thin films only one structure may dominate the bulk of the film. In sputtered thin films of the copolymer, ${ }^{42}$ the orientation of the films depends on the thickness. For such thin films (less than $6 \mathrm{ML}$ ) the chains lie normal to the film surface. As the thickness increases, the chains disorder, until they finally lay flat on the surface. Our LB-grown films show no evidence for this behavior - the X-ray structure suggests that, for all thicknesses ranging from $2 \mathrm{ML}$ up to $150 \mathrm{ML}$, the chains lie parallel to the surface.

The possible bulk crystal structures postulated for the $\mathrm{P}(\mathrm{VDF}-\mathrm{TrFE})$ copolymer in the ferroelectric phase include monoclinic, orthorhombic and distorted hexagonal. ${ }^{41}$ Our data on the surface structure does not provide a conclusive resolution to this controversy, but the LEED and bandstructure results are consistent with the monoclinic bulk structure proposed.

The surface structure, probed using scanning tunneling microscopy, low-energy electron diffraction (Fig. 4) and conduction-band structure mapping (Fig. 7), is distinct from the bulk. There is evidence for relaxation of the first sublayer toward the surface. The resultant surface primitive cell of the copolymer films $\mathrm{P}(\mathrm{VDF}-\mathrm{TrFE}, 70: 30)$ consists of eight monomers and its size is about $5 \times 18 \AA^{2}$. This is consistent with the postulated all-trans structure, with a "zigzag", in the projection of the polymer chain onto the (010) plane from a $13^{\circ}$ deviation $\left( \pm 7^{\circ}\right)$ from the polymer chain line caused by steric hindrance between fluorines. ${ }^{13,43}$ The doubled periodic lattice constant along the chain could come from such a zigzag chain structure and the repeated periodic distance along the chain would be not 2.5 but $5.0 \AA$. With the layer-by-layer growth of the film, the misfit in the placement of the chain to chain placement in the surface results in a superperiodicity from layer to layer in the bulk. The difference in the visualization of this chain to chain displacement in the surface versus the bulk is a consequence of the surface sublayer relaxation.

Structural conformation changes, across the surface ferroelectric phase transition, are also consistent with the observed changes in the angle-resolved core-level photoemission and the work function. The change in structural conformation is accompanied by shortening of the conduction-band dispersion period and shifting of the lowest unoccupied molecular orbital states toward the Fermi level in $\mathrm{P}(\mathrm{VDF}-\mathrm{TrFE})$, as shown in Fig. 7. The shift of the lowest unoccupied molecular orbital states was also observed across the surface phase transition in previous work. ${ }^{8}$

Well above the surface transition temperature, the diminishing resolution of the Brillouin-zone dispersion periodicity and the large shifting of the lowest unoccupied molecular orbital are an indication of increasing structural disorder, as 
indicated in Fig. 9. The loss of the Brillouin-zone periodicity with increasing energy is strongly suggestive of a low activation barrier to rotation and a low Debye temperature.

A surface phase transition $\left(\sim 20^{\circ} \mathrm{C}\right)$ distinct from the bulk phase transition $\left(\sim 80^{\circ} \mathrm{C}\right)$, can be understood partly as a consequence of the distinct surface crystal structure compared to the bulk structure. The surface also has smaller coordination number and a lower effective Debye temperature, though indeed both are very low (about $26 \mathrm{~K}$ for the surface ${ }^{8}$ and 53-67 $\mathrm{K}$ for the bulk ${ }^{44}$ ). The coupling of the electronic structure to the conformational structure could be accompanied by a dynamic Jahn-Teller distortion which was observed through the valence band spitting of the Na-doped 5-ML films of P(VDF-TrFE) with increasing temperature. $^{44,45}$

\section{CONCLUSION}

The bulk crystal structure of the Langmuir-Blodgett films, both a few monolayers and many monolayers thick, is qualitatively similar to spun or melt-drawn films. LangmuirBlodgett films of poly vinylidene fluoride-trifluoroethylene
$\mathrm{P}(\mathrm{VDF}-\mathrm{TrFE})$, copolymer films as thin as $5 \mathrm{ML}$ have qualitatively the same bulk layer structure as thicker films. The surface structure of these Langmuir-Blodgett films is, however, distinct from the bulk structure and there is a surface phase transition $\left(\sim 20^{\circ} \mathrm{C}\right)$ which is distinct from the bulk phase transition $\left(\sim 80^{\circ} \mathrm{C}\right)$. The surface sublayer of $\mathrm{P}$ (VDFTrFE) films is believed to be relaxed toward the surface.

\section{ACKNOWLEDGMENTS}

This work was supported by the Office of Naval Research, the National Science Foundation, and AFOSR Contract No. F30602-98-C-0105 to the W. M. Keck Center for Molecular Electronics, subcontract No. 3531141, and the Nebraska Research Initiative. S.P.P., S.G.Y., and N.N.P. were supported by the Russian Fund for Basic Research (RFBR) through Grant Nos. N99-02-16484 and N98-0217071 and the Inco-Copernicus Program. The authors would like to thank Carol Thompson for useful discussions and Andrew Payzant (ORNL) for his assistance in taking some of the $\mathrm{x}$-ray data.
${ }^{1}$ E. Tosatti, in Electronic Surface and Interface States on Metallic Systems, edited by E. Bertel and M. Donath (World Scientific, Singapore, 1995) p. 67; E. Wimmer, H. Krakauer, M. Weinert, and A. J. Freeman, Phys. Rev. B 24, 864 (1981).

${ }^{2}$ J. W. Chung, in Electronic Surface and Interface States on Metallic Systems, (Ref. 1), p. 127; A. Fasolino and E. Tosatti, Phys. Rev. B 35, 4264 (1987); A. Fasolino, G. Santoro, and E. Tosatti, Surf. Sci. 125, 317 (1983); K. E. Smith and S. D. Kevan, Phys. Rev. B 43, 3986 (1991); 45, 13642 (1992).

${ }^{3}$ T. E. Felter, R. A. Barker, and P. J. Estrup, Phys. Rev. Lett. 38, 1138 (1977).

${ }^{4}$ J. W. Chung, K. S. Shin, and S. C. Houg, Mod. Phys. Lett. B 7, 865 (1993); M. K. Debe and David A. King, Phys. Rev. Lett. 39, 708 (1977); M. Posternak, H. Krakauer, A. J. Freeman, and D. D. Koeling, Phys. Rev. B 21, 5601 (1980); J. C. Comjozano, J. E. Ingksfield, D. A. King, and C. Somertou, J. Phys. C 14, 3099 (1981); D. Singe and H. Krakauer, Phys. Rev. B 37, 3999 (1988); K. E. Smith, G. S. Elliot, and S. D. Kevan, ibid. 42, 5385 (1990).

${ }^{5}$ T. McAvoy, J. Zhang, C. Waldfried, D. N. McIlroy, P. A. Dowben, O. Zeybek, T. Bertrams, and S. D. Barrett, Euro. Phys. J. B (to be published).

${ }^{6}$ J. R. Brews, Phys. Rev. Lett. 18, 662 (1967).

${ }^{7}$ A. V. Bune, V. M. Fridkin, Stephen Ducharme, L. M. Blinov, S. P. Palto, A. V. Sorokin, S. G. Yudin, and A. Zlatkin, Nature (London) 391, 874 (1998).

${ }^{8}$ Jaewu Choi, P. A. Dowben, Shawn Pebley, A. V. Bune, Stephen Ducharme, V. M. Fridkin, S. P. Palto, and N. Petukhova, Phys. Rev. Lett. 80, 1328 (1998).

${ }^{9}$ Jaewu Choi, P. A. Dowben, Stephen Ducharme, V. M. Fridkin, S. P. Palto, N. Petukhova, and S. G. Yudin, Phys. Lett. A 249, 505 (1998).

${ }^{10}$ P. A. Dowben, Philos. Trans. R. Soc. London, Ser. A 356, 267 (1998)

${ }^{11}$ S. D. Kevan and N. G. Stoffel, Phys. Rev. Lett. 53, 702 (1984); S.
D. Kevan, Phys. Rev. B 32, 2344 (1985); R. M. Feenstra and M. A. Lutz, 42, 5391 (1990); Luca Gavioli, Maria Grazia Betti, and Carlo Mariani, Phys. Rev. Lett. 77, 3869 (1996).

${ }^{12}$ Takeo Furukawa, Phase Transit. 18, 143 (1989).

${ }^{13} \mathrm{G}$. Thomas Davis, in The Applications of Ferroelectric Polymers, edited by T. T. Wang, J. M. Herbert, and A. M. Glass (Bell \& Bain, Glasgow, 1988), Chap. 9, p. 37.

${ }^{14}$ J. F. Legrand, Ferroelectrics 91, 303 (1989).

${ }^{15}$ K. Tashiro, R. Tanaka, K. Ushitora, and M. Kobayashi, Ferroelectrics 171, 145 (1995).

${ }^{16}$ C. K. Purvis and P. L. Tayor, J. Appl. Phys. 54, 1021 (1983).

${ }^{17}$ K. Tashiro, Y. Abe, and M. Kobayashi, Ferroelectrics 171, 281 (1995), and reference therein.

${ }^{18}$ R. Al-Jishi and P. L. Taylor, J. Appl. Phys. 57, 902 (1985).

${ }^{19}$ N. C. Banik, F. P. Boyle, T. J. Sluckin, P. L. Taylor, S. K. Tripathy, and A. J. Hopfinger, J. Chem. Phys. 72, 3191 (1986).

${ }^{20}$ M. A. Bachmann and J. B. Lando, Macromolecules 14, 40 (1981).

${ }^{21}$ S. Weinhold, M. H. Litt, and J. B. Lando, Macromolecules 13, 1178 (1980).

${ }^{22}$ Andrew J. Lovinger, Macromolecules 18, 910 (1985).

${ }^{23}$ S. Palto, L. Blinov, A. Bune, E. Dubovik, V. Fridkin, N. Petukhova, K. Verkhovsakya, and S. Yudin, Ferroelectr. Lett. Sect. 19, 65 (1995).

${ }^{24}$ L. M. Blinov, Usp. Fiz. Nauk 155, 443 (1988) [Sov. Phys. Usp. 31, 623 (1988)].

${ }^{25}$ J. H. Scofield, J. Electron Spectrosc. Relat. Phenom. 8, 129 (1976).

${ }^{26}$ P. W. Erdman and E. C. Zipf, Rev. Sci. Instrum. 53, 225 (1982).

${ }^{27}$ V. Dose, Appl. Phys. 14, 117 (1977).

${ }^{28}$ K. J. Kim, G. B. Kim, C. L. Vanlencia, and J. F. Rabolt, J. Polym. Sci. Part B. Polym. Phys. 32, 2435 (1994).

${ }^{29}$ Andrew J. Lovinger, Science 220, 1115 (1983).

${ }^{30}$ K. Toshira, K. Takano, M. Kobayashi, Y. Chatani, and H. Tadokoro, Ferroelectrics 57, 297 (1984). 
${ }^{31}$ P. J. Estrup and E. G. McRae, Surf. Sci. 25, 1 (1971).

${ }^{32}$ Keiji Morokuma, J. Chem. Phys. 54, 962 (1971).

${ }^{33}$ Alfred Karpfen, J. Chem. Phys. 75, 238 (1981).

${ }^{34}$ János J. Ladik, in Quantum Theory of Polymers as Solid (Plenum, New York, 1988), Chap. 2, p. 53.

${ }^{35}$ P. Otto, J. Ladik, and W. Förner, Chem. Phys. 95, 365 (1985).

${ }^{36}$ M. P. Seah and W. A. Dench, Surf. Sci. 83, 391 (1979).

${ }^{37}$ Jaewu Choi, C. Waldfried, S.-H. Liou, and P. A. Dowben, J. Vac. Sci. Technol. A 16, 2950 (1998); Jaewu Choi, Jiandi Zhang, S.-H. Liou, P. A. Dowben, and E. W. Plummer, Phys. Rev. B 59, 1819 (1999).

${ }^{38}$ D. Briggs and M. P. Seah, Practical Surface Analysis: Vol. 1: Auger and X-ray Photoelectron Spectroscopy, 2nd ed. (Wiley, New York, 1990).

${ }^{39}$ A. Bune, Stephen Ducharme, V. Fridkin, L. Blinov, S. Palto, N.
Petukova, and S. Yudin, Appl. Phys. Lett. 67, 25 (1995).

${ }^{40}$ Ferroelectric Polymers, edited by Kohji Tashiro, H. S. Nalwa, (Marcel Dekker, New York, 1995), Chap. 2, pp. 63-181.

${ }^{41}$ M. V. Fernandez, A. Suzuki, and A. Chiba, Macromolecules 20, 1806 (1987).

${ }^{42}$ K. Tashiro, K. Yamamato, M. Kobayashi, and O. Phaovibul, Rep. Prog. Polym. Phys. Jpn. 30, 123 (1987).

${ }^{43}$ R. Hasegawa, T. Takahashi, Y. Chatani, and H. Tadakoro, Polymer J. 3, 600 (1972).

${ }^{44}$ C. N. Borca, Jaewu Choi, Shireen Adenwalla, Stephen Ducharme, P. A. Dowben, Lee Robertson, V. M. Fridkin, S. P. Palto, and N. Petukhova, Appl. Phys. Lett. 74, 347 (1999).

${ }^{45}$ Jaewu Choi, P. A. Dowben, C. N. Borca, Shireen Adenwalla, A. V. Bune, Stephen Ducharme, V. M. Fridkin, S. P. Palto, and N. Petuhova, Phys. Rev. B 59, 1819 (1999). 